\title{
Neurociencia y eneagrama: Reconfigurando los equipos de trabajo
}

\section{Neuroscience and enneagram: Reconfiguring work teams}

\author{
LINAREZ-PLACENCIA, Gildardo†*, ESPINOZA-CASTELO, Luz Maria y PIMENTEL-FÉLIX, Ana \\ Fabiola
}

Universidad Tecnológica de San Luis Río Colorado, Coordinación de academias, San Luis Río Colorado, Sonora, México.

ID 1 ${ }^{\mathrm{er}}$ Autor: Gildardo, Linarez-Placencia / ORC ID: 0000-0002-4711-676X, CVU CONACYT ID: 669198

ID $1^{\mathrm{er}}$ Coautor: Luz María, Espinoza-Castelo / ORC ID: 0000-0001-9449-2963, CVU CONACYT ID: 278365

ID $2^{\text {do }}$ Coautor: Ana Fabiola, Pimentel-Félix / ORC ID: 0000-0002-0021-715X, CVU CONACYT ID: 1013511

DOI: $10.35429 /$ JCP.2019.8.3.6.16

Recibido 16 de Marzo, 2019; Aceptado 30 de Junio, 2019

\section{Resumen}

La competitividad es fuero de la modernidad en todos los sectores de la sociedad. La ejecución de tareas se ha vuelto demasiado compleja por los problemas que ha traído la integración de la globalización como sinónimo de competencia. Indubitablemente, la forma de responder a la complejidad del entorno actual es por medio de la integración de equipos de trabajo que puedan ser eficientes; y la única forma de garantizar el éxito es mediante la supletoriedad de los rasgos de la personalidad pocos desarrollados con las habilidades bien trabajadas de otros miembros del equipo. Por lo anterior, la presente investigación desarrolló un estudio cuasi-experimental en 62 personas; para demostrar que los equipos de trabajo formados por los eneatipos o rasgos de la personalidad, obtenidos por la prueba del mapa del eneagrama, son más eficientes que los equipos tradicionales. Las principales aportaciones al vacío del conocimiento de esta investigación son: la desmitificación del eneagrama; demostrando mediante un estudio cuantitativo que los participantes obtienen mejores resultados cuando se trabaja en equipos formados con eneagrama; los participantes tienen una percepción positiva sobre eneagrama; y utilizando las herramientas de la neurociencia se prueba con datos fisiológicos que los preceptos teóricos de eneagrama son correctos.

Equipos de trabajo, Capital humano, Eneagrama, Neurociencia

\begin{abstract}
Competitiveness is outside modernity in all sectors of society. The execution of tasks has become too complex due to the problems brought by the integration of globalization as a synonym for competition. Undoubtedly, the way to respond to the complexity of the current environment is through the integration of work teams that can be efficient; And the only way to guarantee success is by supplementing the few personality traits developed with the well-worked skills of other team members. Therefore, this research developed a quasi-experimental study in 62 people; to demonstrate that work teams formed by eneatypes or personality traits, obtained by the enneagram map test, are more efficient than traditional equipment. The main contributions to the knowledge gap of this research are: demystification of the enneagram; demonstrating through a quantitative study that the participants obtain better results when working in teams formed with enneagram; participants have a positive perception about enneagram; and using the tools of neuroscience it is proved with physiological data that the theoretical precepts of enneagram are correct.
\end{abstract}

Work teams, Human capital, Enneagram, Neuroscience

Citación: LINAREZ-PLACENCIA, Gildardo, ESPINOZA-CASTELO, Luz Maria y PIMENTEL-FÉLIX, Ana Fabiola. Neurociencia y eneagrama: Reconfigurando los equipos de trabajo. Revista de Pedagogía Crítica. 2019, 3-8: 6-16

\footnotetext{
* Correspondencia al Autor (Correo electrónico: glinarez@ hotmail.com)

$\dagger$ Investigador contribuyendo como primer autor.
} 


\section{Introducción}

Vivir en el siglo de la innovación y de la creatividad ha conducido a la sociedad moderna a la era de la competitividad. La industria, servicios, comercio y todos los sectores económicos y sociales tienen como objetivo lograr las mejores expectativas financieras, para intentar sobrevivir en un mundo que parece ser tierra de nadie y en donde el error de las malas decisiones no tiene cabida (Molina, Israel, \& Ampudia Sjogreen, 2018). Por lo anterior, el desarrollo del capital humano se convierte en una necesidad para afrontar los retos implícitos de la competitividad, en virtud de que es menester exclusivo del recurso humano la toma de decisiones.

Indubitablemente que la competitividad actual pone de manifiesto la complejidad de las tareas laborales, académicas, sociales y cotidianas; es decir, toda actividad en la que se desenvuelva el hombre requiere de transitar del individualismo al trabajo colectivo. En ese sentido, para Mascareño (2018) toda acción del hombre "ha adquirido robustez, extensión y periodicidad sin precedentes en la evolución social" (p. 111), lo que confirma la obligatoriedad de realizar el trabajo en equipo como una vía para la solución de los problemas de la modernidad.

A partir del axioma del trabajo en equipo nacen los rizomas relativos a las metodologías para la integración correcta de equipos de trabajo. A pesar que la naturaleza del hombre tiende a ser un ente social (Hernández, Armijo, \& Sánchez, 2019) capaz de integrarse en comunidades y grupos; sin embargo, ese proceso natural no está alineado a los principios de eficiencia y pertinencia como su eje rector, sino que el hombre se junta por otros factores subjetivos como pudieran ser: familia, religión, zona geográfica y apariencia física.

Es entonces que la revisión de las formas y factores de integración de equipos de trabajo adquiere una relevancia mayor por el mínimo margen de error que exige la competitividad de la era actual; por ello, nace el eneagrama como una forma de analizar y clasificar los factores importantes de los rasgos de la personalidad como herramientas para formar equipos de trabajo.
Pese a la necesidad existente, el eneagrama ha sido satanizado por su supuesto origen en la astrología (López-Cantos \& Millán Yeste, 2018), asimismo que es asociado con cuestiones espirituales demeritando su verdadero valor.

Por ello, el presente trabajo buscar demostrar la factibilidad del eneagrama para la conformación de cualquier equipo de trabajo mediante un estudio de tareas de ejecución en equipo, que será reafirmado mediante el uso de la neurociencia para demostrar que sus preceptos teóricos son comprobables desde mediciones cuantitativas a nivel cerebral del acoplamiento de las emociones en el trabajo en conjunto

\section{Eneagrama y neurociencia; equipos de trabajo exitosos}

\section{Eneagrama}

Se aborda el estudio del eneagrama desde el punto de vista del comportamiento de la persona a nivel de los rasgos de la personalidad, para encontrar los puntos de una conexión más eficiente de los elementos requeridos para lograr el trabajo en equipo. Por lo que, para atender su significado y evitar caer en la deriva de la subjetividad, es necesario definirlo como punto de partida del presente análisis:

El eneagrama de la personalidad es una herramienta psicológica utilizada con la finalidad de conocer la psique humana, así como comprender la personalidad de los individuos y la manera en que ésta les condiciona a la hora de vivir, con el fin de desarrollar al máximo el potencial humano (Ramírez Villanueva, 2018, p. 19).

Con la anterior definición se elimina por completo y de tajo la errónea idea del eneagrama como un elemento esotérico, en virtud de que responde al estudio de la psique del hombre. La anterior confusión se fundamentó en el solo hecho de utilizar como base una estrella de 9 puntos, misma que es utilizada para otros fines.

Asimismo y atendiendo a la etimología de la palabra, eneagrama tiene su origen en el griego. Es un vocablo que se compone de dos términos: "enea, que significa nueve y grama que es gráfica; es decir, gráfica de nueve puntos" (Vargas, 2015, p. 11) 
Cada punto geográfico que ofrece el mapa del eneagrama se refiere a tipos de personalidades, que también son conocidos como eneatipos, y estos sirven para identificar a cada miembro de un posible equipo de trabajo; además sirve como referencia para conocer por qué cada persona reacciona e interpreta la realidad de manera distinta; también son útiles para la aplicación de estilos de liderazgo; y, por último, preparar equipos de trabajo afines a las fortalezas de cada líder(Castro Castillo, 2019).

\section{Eneatipos.}

Eneatipo 1. El perfeccionista.

Los rasgos de esta personalidad se definen por el rigor del pensamiento único como vía para hacer las cosas. Suelen ser cerrados y viscerales, tal vez marcados por una infancia dificil $y$, por ende, desarrollados bajo los castigos. En virtud de lo anterior, la disciplina y cuidado de la ejecución de las tareas es su principal virtud; en contrario, tienen la desventaja en su elevado nivel de exigencia lo cual les puede ocasionar ira y frustración (Fernández, 2010).

Eneatipo 2. El ayudador.

Los rasgos básicos del perfil del este eneatipo se definen por la capacidad de sentir empatía con los demás, además ser serviciales y acomedidos. Su principal ventaja en el trabajo en equipo es su espíritu de cooperación; y su desventaja es que pueden ser posesivos en cuanto a las tareas y manipuladores con tal sentirse útiles (Riso, 1993).

\section{Eneatipo 3. El triunfador.}

Su esencia es buscar el éxito dentro del trabajo en equipo, por lo tanto, busca el reconocimiento del grupo mediante el trabajo operativo competente, es comprometido y es parte esencial que todo equipo debería de tener, pero esa búsqueda incesante del éxito puede ser por simple alimento del ego y caer en el engaño de un falso compromiso (Vargas, 2015).

Eneatipo 4 El melancólico.

Este tipo de eneagrama se considera que es romántico por naturaleza y sensible, lo cual puede ser demasiado útil en el trabajo en equipo en temas sociales y de apoyo a las necesidades sociales.
Cuando se necesitan ideas sobre estética, belleza y arte son ideales para hacer ese trabajo. Su principal desventaja es que sienten miedo al rechazo de su trabajo (Beesing, Nogosek, \& O'Leary, 1992).

\section{Eneatipo $5 \mathrm{El}$ investigador.}

El trabajo analítico es el principal aporte del investigador al equipo. Es indagador por naturaleza, curioso, independiente y solitario. $\mathrm{Su}$ principal desventaja es la integración al equipo ya que con frecuencia pude sentirse invadido en su espacio y trabajo por los demás integrantes del equipo (Beesing et al., 1992).

\section{Eneatipo 6. Leal}

Su trabajo en el equipo se basa en la fidelidad hacia los objetivos y tareas, muestra respeto por la responsabilidad y sabe trabajar bajo discreción absoluta. Es decir, este tipo de personas son en las que puedes confiar. Su principal desventaja es miedo a no ser correspondido en la lealtad (Vargas, 2015).

\section{Eneatipo 7. Entusiasta}

Este tipo de personalidad le aporta ideas brillantes al trabajo en equipo, siempre se muestra cooperativo y decidido a trabajar por el equipo en nuevos proyectos para la consecución de las metas, pero su miedo al sufrimiento lo frena a comprometerse a fondo con el equipo de trabajo (Vargas, 2015).

\section{Eneatipo 8. El líder}

Se definen por su capacidad para convencer mediante el uso de la comunicación y trabajo coordinado, siendo capaces de delegar trabajo y compartir responsabilidades, mismas que son cualidades esenciales para todo el trabajo en equipo; sin embargo, como características negativas debido a su grado de compromiso pueden ser muy combativos y destructivos en el equipo de trabajo(Riso, 1993).

Eneatipo 9. El mediador.

Los rasgos de su personalidad se definen por la capacidad de hacer suyas las ideas de los demás, porque tiene la posibilidad de hacer a un lado sus pasiones con tal de lograr acuerdos. 
Su principal ventaja es que busca dentro de los equipos de trabajo la armonía y buen trato; misma situación que se convierte en su desventaja al tratar de evitar los conflictos y solo disfrazarlos mostrando una tendencia a postergar la solución de estos(Fernández, 2010).

\section{Las triadas}

Para el correcto funcionamiento de un equipo de trabajo se tienen que conformar, según recomendaciones de los diversos autores mencionados en este trabajo, en una combinación de tres tipos de eneatipos para formar triadas que complementen sus ventajas con las desventajas del otro tipo de participantes, y permitan engrandecer el equipo humano.

1. Triada del instinto. "El cuerpo o inteligencia instintiva: Engloba la fuerza, el autocontrol, los instintos" (Delgado, 2014, p. 18). Igualmente se asocia con la decisiones viscerales, intuitivas y por el espíritu de sobrevivencia, es el lado totalmente irracional que se forma con los eneatipos 8-9-1.

2. Triada del pensamiento. Es la parte lógica y de raciocinio que caracteriza y distingue al hombre de los demás animales por el esquema estructurado para generar pensamientos y tomar decisiones centradas en obtener las mejores posibilidades. Esta conformado con los eneatipos 7-6-5.

3. Triada de las emociones. Se basa en la capacidad de distinguir las emociones, los sentimientos que posee cada individuo. En un equipo de trabajo se refiere a la capacidad de ser sensible y de generar ideas pensadas en los momentos y situaciones particulares de cada contexto. Forman parte de esta triada los eneatipos 4-3-2.

\section{Neurociencia}

Se observa que para el hombre siempre ha sido una gran interrogante el conocer cómo funciona la máquina que se tiene sobre los hombros, porque es un sistema nervioso complejo y maravilloso capaz de producir ideas de forma sistemática, que son la base para lograr la transformación de la propia historia del ser humano.
Dicha preocupación y ocupación no es de la actualidad, sino que desde tiempos inmemoriales se ha buscado la forma de saber como trabaja el cerebro. Por ejemplo, al principio de la historia se hacían trepanaciones para conocer el proceso de generación del pensamiento.

En la medida en que las herramientas de las cuales dispone el hombre se fueron tecnificando se empezó a conocer más el cerebro. En lo referente a ese proceso se fueron creando muchas teorías sin un sustento o demostración de su fundamentación, pero en la medida que se ha podido se van destruyendo mitos con el uso de la neurociencia.

La neurociencia tiene como finalidad conocer el funcionamiento de nuestro cerebro para poder explicar de una forma demostrativa los sentimientos y emociones (Campos \& Álvarez, 2019). Es así que la neurociencia, para ser considerada tal, tiene que explicar y demostrar lo que se dice mediante el uso de sensores, medidores de frencuencias, cascos encefalográficos o cualquier dispositivo de medición asociado al cerebro o al funcionamiento de los sentidos.

La neurociencia dio un giro importante en la historia con la aparición de la la teoría celular del cerebro, establecida por Schwan. Con base en lo anterior, se formularon dos teorías: la primera, la teoría celular de Golgi que estaba basada en la idea de que las neuronas eran un retículo que las hacía muy difícil de diferenciar una de otra porque se encontraban unidas por axones y dendritas; y la segunda, teoría neuronal de Ramón y Cajal que sostenía que las neuronas se diferenciaban, estableciendo que existía un tipo de contactos entre ellas llamado sinapsis (López Muñoz et al., 2008).

\section{Instrumentos de medición de la neurociencia}

Como ya se mencionó y ahora se reafirma, una parte importante para su correcto desarrollo de la neurociencia es el contar con instrumentos de medición de la actividad cerebral y de otras formas que permitan llegar al proceso de generación de ideas; por lo tanto, se procede a realizar un análisis de las diferentes herramientas con las que se cuentan en la actualidad. 
Dentro de las herramientas más populares para el desarrollo de la neurociencia son la electroencefalografía (EEG) y la Resonancia Magnética Funcional. Y generalmente son combinadas con otras mediciones biométricas, como puedan ser la medición de la respuesta galvánica de la piel, seguimiento ocular (eyetracking), medición del ritmo cardiaco o la electromiografía (Benito, 2011).

La mejor manera de obtener información de las personas y de las formas en las que se comportan en los equipos de trabajo es mediante la maganetoencefalografía, que utiliza aparatos modernos que de una forma no invasiva y en tiempo real muestran mapas completos de la actividad cerebral y de las regiones activas y apagadas al momento de hacer la toma de decisiones (Braidot, 2013).

Existen otros instrumentos de medición de la resistencia de la piel a los estímulos externos en el procreso de trabajo en equipo, que mediante un flujo constante de energía eléctrica se puede medir las variaciones de la resistencia a cambios emocionales de grupos sociales llamado sociographf (Aiger, Palacín, \& Cornejo, 2013).

Según los estudios de Rúas-Araújo, Punín Larrea, Gómez Alvarado, CuestaMorales, y Ratté, (2015), han demostrado que la actividad cerebral se refleja en las emociones, mismas que pueden ser analizadas según estándares de reconocimiento facial. Por lo tanto, las emociones como la alegría, tristeza, miedo, asco, desprecio, sorpresa e ira pueden reflejarse en el rostro de una persona. Cabe destacar que el protocolo de Ekman y Freisser comprueba que la gesticulación en el rostro funciona en distintos países y culturas, que mantienen el mismo comportamiento ante las emociones descritas con anterioridad, por lo que su uso puede generalizarse en cualquier ambiente o culutura.

Para el desarrollo de la presente investigación se utilizó un casco tipo encefalograma para conocer las emociones y sentimientos que los integrantes del equipo estaban sintiendo en tiempo real; además, se trabajó con un software de reconocimiento facial para detectar las emociones de las personas; asimismo, se utilizó un reloj capaz de monitorear los cambios de temperatura, pulsaciones y ritmo cardiaco de los participantes.

ISSN 2523-2479

ECORFAN $^{\circledR}$ Todos los derechos reservados
Las anteriores tres herramientas se utilizaron con el fin de cumplir con la función demostrativa de la neurociencia y, sobre todo, deerminar la funcionalidad del eneagrama como herramienta para la conformación de equipos de trabajo.

Los principales protagonistas del éxito de los equipos de trabajo son las ideas, la mente y la conducta humana (Barthelemy \& Rodriguez, 2016). Por ello, se convierte en un eje primordial el estudio del cerebro para el correcto funcionamiento de los equipos de trabajo y del capital humano en cualquier tipo de organización, ya que en el comportamiento se concentran esas variables y dan como resultado las acciones y decisiones del capital humano en una organización.

Como se puede observar, la neurociencia debe ser una palanca que favorezca el desarrollo de la formación de equipos de trabajo y del desarrollo del capital humano; para que se pueda responder a todos los retos de la modernidad empresarial, los cuales son bastante complejos. Cabe destacar, que la complejidad de la problemática de la administración solo se va a solucionar con aportes científicos que den una base sólida al desarrollo de la ciencia administrativa mediante la conformación de verdaderos equipos de trabajo eficientes.

\section{Equipos de trabajo}

La necesidad de estar a la vanguardia en el mundo de la competitividad obliga a las empresas y organizaciones a realizar trabajo en equipo (Villegas \& Sánchez, 2018), por ende, el diseño de los equipos de trabajo debe ser una tarea realizada a consciencia que involucre el estudio de los rasgos de la personalidad de los integrantes, mediante el uso del eneagrama y neurociencias.

En ese sentido, no solamente se deben de evaluar a los nuevos integrantes de los equipos de trabajo, sino que se convierte en una obligación el evaluar a los elementos internos de un equipo ya existentes; por ello, la metacognición juega un papel importante para saber los eneatipos que ya se tienen y cual es el complemento perfecto para lograr un trabajo en equipo eficiente. 
Es decir, las organizaciones que entienden como trabajar coordinadamente no buscan a los elementos o personas más preparadas u organizadas, ya que se basan la mayoria de las veces en lo que tienen y solo buscan los complementos perfectos para lograr cumplimentar los objetivos organizacionales.

Entonces, los equipos de alto rendimiento son aquellos que se forman tomando en cuenta las triadas del eneagrama. Razón por la cual todas las organizaciones deben de utilizar los test que existen para determinar los eneatipos de su capital humano y las demás herramientas de la neurociencia para corrobar que el estado emocional es adecuado. Así que la inteligencia emocional es un factor de éxito en el liderazgo de las organizaciones, y es mediante el eneagrama y la neurociencia el camino más corto para lograrlo.

\section{Las triadas y los tres cerebros}

El cerebro del hombre se compone de tres capas llamadas: reptiliana, límbica y neocórtex. Para entender mejor su funcionamiento se convierte en una obligación definir cada una de ellas:

La parte reptiliana o cerebro primitivo es la parte del inconsciente y se asocia con la toma de decisiones basadas en el miedo y los instintos de supervivencia; la segunda parte, es la que se encarga de las emociones en el hombres, su función límbica es parte del subconsciente que se ha demostrado está asociada o desata la parte del cerebro reptiliano; y la tercer capa, que es la encargada del proceso se razonamiento del hombre, es decir, lo consciente (Requena, 2005).

En ese sentido se puede observar una clara asociación de los eneatipos 8-9-1 llamados como la triada del instinto con el funcionamiento del cerebro reptiliano. Asimismo, se observa que los eneatipos 4-3-2 que forman la triada de las emociones, pueden ser asociados y comprobar su funcionamiento con el cerebro límbico.

Y, por último, los eneatipos 7-6-5 que tienen comportamientos racionales son clasificados dentro del funcionamiento del cerebro neocórtex que se describió con anterioridad.
Con lo anterior, queda de manifiesto la posibilidad que durante el experimento se monitoree la actividad cerebral a fin de comprobar que los eneatipos de la personalidad están bien definidos y, por ende, abonan en gran medida al trabajo en equipo.

\section{Metodología}

Se realizó un estudio cuasiexperimental para determinar si los conceptos del eneagrama y su respectiva comprobación mediante neurociencia surten efecto en la realidad para el trabajo en equipo. Mediante un ejercicio práctico con estudiantes de la licenciatura en Desarrollo de negocios se integraron equipos considerando las triadas en la búsqueda de medir la eficiencia del trabajo en cuanto al tiempo que se realizó determinada actividad. Para eliminar el efecto de la experiencia en cuanto a la actividad realizada se cruzaron dos grupos, poniendo primero a un grupo a realizar la actividad mediante la integración de equipos por afinidad y por eneagrama; y viceversa con el otro grupo, para tener puntos de comparación entre el trabajo realizado.

Además, al terminar el trabajo en equipo se les aplicó un cuestionario a los integrantes del equipo para conocer su percepción sobre el trabajo en equipo con eneagrama y recabar su sentir sobre el éxito de la integración de los equipos de trabajo mediante las triadas de eneagrama.

Por último, se utilizaron herramientas de neurociencia para detectar las emociones en tiempo real cuando se realizaba la actividad en equipo, buscando coincidencias con las teorías y la correcta integración de las triadas. Mediante estas herramientas se busca eliminar la subjetividad de los cuestionarios aplicados a los participantes en el trabajo en equipo.

Como ya se mencionó los principios de la neurociencia, eneagrama y trabajo en equipo son de aplicación universal, por lo que trabajar con estudiantes equivaldría a trabajar con cualquier grupo de personas integradas en un equipo de trabajo formal e informal. 


\section{Análisis y discusión de resultados}

Dentro del primer grupo de análisis se realizaron la aplicación de 62 cuestionarios a sujetos participantes en la ejecución de una tarea en equipo para evaluar la efectividad de la conformación del eneagrama de acuerdo a las triadas mencionadas, obteniéndose los siguientes resultados:

\begin{tabular}{|c|c|c|c|c|c|}
\hline & Frecuencia & $\%$ & \% válido & $\%$ acum \\
\hline \multirow[t]{3}{*}{ Válido } & Sí & 46 & 74.2 & 74.2 & 74.2 \\
\hline & No & 16 & 25.8 & 25.8 & 100.0 \\
\hline & Total & 62 & 100.0 & 100.0 & \\
\hline
\end{tabular}

Tabla 1 Te gustó trabajar en equipo con eneagrama Fuente: Elaboración Propia

Se observa que el $74.2 \%$ de los participantes está de acuerdo en la forma con la que se asignaron los equipos de trabajo mediante el mapa de eneatipos. Es decir, desde la perspectiva del participante el eneagrama fue funcional para la integración de equipos de trabajos eficientes, se sintieron cómodos y, por lo tanto, estan dispuestos a trabajar en ese equipo. Mientras solo el $25.8 \%$ no está de acuerdo en la conformación de equipos de trabajo.

\begin{tabular}{|l|l|r|r|r|r|}
\multicolumn{2}{c}{} & Frecuencia & $\%$ & $\%$ válido $\%$ acum. \\
\hline \multirow{3}{*}{ Válido } & Sí & 42 & 67.7 & 67.7 & 67.7 \\
\cline { 2 - 6 } & No & 20 & 32.3 & 32.3 & 100.0 \\
\cline { 2 - 6 } & Total & 62 & 100.0 & 100.0 & \\
\hline
\end{tabular}

Tabla 2 ¿Habías considerado trabajar con los integrantes de tu equipo según en eneagrama?

Fuente: Elaboración Propia

El $67.7 \%$ de los participantes en el diseño no experimental habían considerado trabajar con los integrantes del equipo formado con el mapa de los eneatipos de eneagrama, mientras que el $32.3 \%$ no lo había considerado. El porcentaje tan alto de que sí lo habían considerado es porque los grupos con los que se trabajo llevan más de un año juntos $\mathrm{y}$, por ello, dan esos resultados.

\begin{tabular}{|c|c|c|c|c|}
\hline & & Frecuencia & $\begin{array}{c}\% \\
\text { válido }\end{array}$ & $\begin{array}{c}\% \\
\text { acum }\end{array}$ \\
\hline \multirow[t]{6}{*}{ Válido } & $\begin{array}{ll}\text { Totalmente } & \text { en } \\
\text { desacuerdo } & \end{array}$ & 5 & 8.1 & 8.1 \\
\hline & En desacuerdo & 6 & 9.7 & 17.7 \\
\hline & Sin opinión & 8 & 12.9 & 30.6 \\
\hline & De acuerdo & 21 & 33.9 & 64.5 \\
\hline & Totalmente de acuerdo & 22 & 35.5 & 100.0 \\
\hline & Total & 62 & 100.0 & \\
\hline
\end{tabular}

Tabla 3 El desempeño de mi equipo es excelente Fuente: Elaboración Propia
El $35.5 \%$ de los participantes tiene la percepción de que el trabajo en equipo con eneagrama fue excelente, más un $33.9 \%$ que está de acuerdo; dan un total del $69.4 \%$ con opinión positiva; mientras el $12.9 \%$ no tiene opinión sobre los buenos resultados del trabajo en equipo con eneagrama; y tan solo el $17.8 \%$ (9.7\% en desacuerdo y 8.1 en total deascuerdo) restante tiene un comentario negativo o está en contra de la afirmación de que los equipos de trabajo con eneagrama son excelentes.

\begin{tabular}{|c|c|c|c|c|c|}
\hline & & Frecuencia & $\%$ & $\begin{array}{c}\% \\
\text { válido }\end{array}$ & $\begin{array}{c}\% \\
\text { acum. }\end{array}$ \\
\hline \multirow[t]{6}{*}{ Válido } & $\begin{array}{l}\text { Totalmente en } \\
\text { desacuerdo }\end{array}$ & 7 & 11.3 & 11.3 & 11.3 \\
\hline & En desacuerdo & 5 & 8.1 & 8.1 & 19.4 \\
\hline & Sin opinión & 7 & 11.3 & 11.3 & 30.6 \\
\hline & De acuerdo & 30 & 48.4 & 48.4 & 79.0 \\
\hline & $\begin{array}{l}\text { Totalmente de } \\
\text { acuerdo }\end{array}$ & 13 & 21.0 & 21.0 & 100.0 \\
\hline & Total & 62 & 100.0 & 100.0 & \\
\hline
\end{tabular}

Tabla 4 Mis características de los rasgos de la personalidad abonan al trabajo en equipo

Fuente: Elaboración Propia

El $21 \%$ de los participantes en equipos de trabajo formados por eneagrama sienten que el trabajo se ve beneficiados porque sienten que sus ventajas y desventajas se complementan perfectamente con las de los otros miembros del equipo y un $48.4 \%$ están de acuerdo; es decir el $69.4 \%$. tienen una opinión positiva. Mientras el $11.3 \%$ no tiene opinión al respecto, y tan solo el $19.4 \%$ (8.1 en desacuerdo y 11.3 en total desacuerdo) tiene una opinión negativa sobre los conformación de equipos de trabajo con eneagrama.

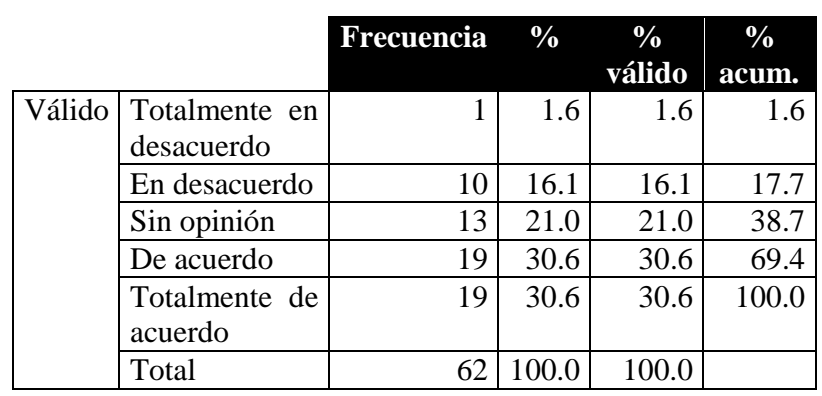

Tabla 5 La comunicación en el equipo con eneagrama fue un factor de éxito

Fuente: Elaboración Propia

Tal y como se mencionó en las referencias teóricas, la comunicación es un factor primordial para que el trabajo en equipo se haga de una forma exitosa. 
En ese sentido un $61.2 \%$ (30.6\% está totalmente de acuerdo y otro $30.6 \%$ de acuerdo) tiene la percepción de que con equipos de trabajo formados por eneagrama la comunicación es efectiva; mientras un $21 \%$ no tiene opinión; y, por último, un $16.1 \%$ está en desacuerdo, mientras un $1.6 \%$ está totalmente en desacuerdo.

\begin{tabular}{|c|l|r|r|r|r|}
\multicolumn{2}{c|}{ Frecuencia } & \multicolumn{2}{c|}{$\%$} & \% válido & \% acum \\
\hline \multirow{4}{*}{ Válido } & Totalmente en desacuerdo & 2 & 3.2 & 3.2 & 3.2 \\
\cline { 2 - 6 } & En desacuerdo & 8 & 12.9 & 12.9 & 16.1 \\
\cline { 2 - 6 } & Sin opinión & 15 & 24.2 & 24.2 & 40.3 \\
\cline { 2 - 6 } & De acuerdo & 24 & 38.7 & 38.7 & 79.0 \\
\cline { 2 - 6 } & Totalmente de acuerdo & 13 & 21.0 & 21.0 & 100.0 \\
\cline { 2 - 6 } & Total & 62 & 100.0 & 100.0 & \\
\hline
\end{tabular}

Tabla 6 El liderazgo en el equipo con eneagrama fue un factor de éxito

Fuente: Elaboración Propia

Es claro que sin liderazgo los equipos de trabajo naufragan y no cumplen con los objetivos propuestos en las organizaciones. Por lo anterior, se cuestionó a los participantes en el experimento sobre el liderazgo natural que debió de haber sucedido en el evento, obteniendose lo siguiente: el $21 \%$ está totalmente de acuerdo en la presencia de un liderazgo, el 38.7 está de acuerdo, es decir, se tiene una percepción positiva sumada de 59.7 $\%$; el $24.2 \%$ no tiene opinión; y el resto 16.1 tiene una percepción negativa, que está conformado por el $12.9 \%$ en desacuerdo y el $3.2 \%$ está totalmente en desacuerdo.

\begin{tabular}{|c|c|c|c|c|c|}
\hline & & Frecuencia & $\%$ & $\begin{array}{c}\% \\
\text { válido }\end{array}$ & $\begin{array}{c}\% \\
\text { Acum }\end{array}$ \\
\hline \multirow[t]{6}{*}{ Válido } & $\begin{array}{ll} & \text { Totalmente } \\
\text { en } & \text { desacuerdo }\end{array}$ & 1 & 1.6 & 1.6 & 1.6 \\
\hline & En desacuerdo & 8 & 12.9 & 12.9 & 14.5 \\
\hline & Sin opinión & 13 & 21.0 & 21.0 & 35.5 \\
\hline & De acuerdo & 24 & 38.7 & 38.7 & 74.2 \\
\hline & $\begin{array}{l}\text { Totalmente de } \\
\text { acuerdo }\end{array}$ & 16 & 25.8 & 25.8 & 100.0 \\
\hline & Total & 62 & 100.0 & 100.0 & \\
\hline
\end{tabular}

Tabla 7 La organización en el equipo con eneagrama fue un factor de éxito

Fuente: Elaboración Propia

La organización es la clave de la estructura y buenos resultados de los equipos de trabajo, por ello, se les cuestionó a los participantes sobre como la organización se veía beneficiada por los miembros que trabajan bajo los rasgos del eneagrama, siendo los resultados los siguientes: el $25.8 \%$ está totalmente de acuerdo, el 38.7 está de acuerdo, el $21 \%$ no tiene opinión, el $12.9 \%$ está en desacuerdo y el $1.6 \%$ en total desacuerdo.
Como se observa la mayoría de los participantes tiene una opinión positiva sobre la organización en los equipos de trabajo con eneagrama.

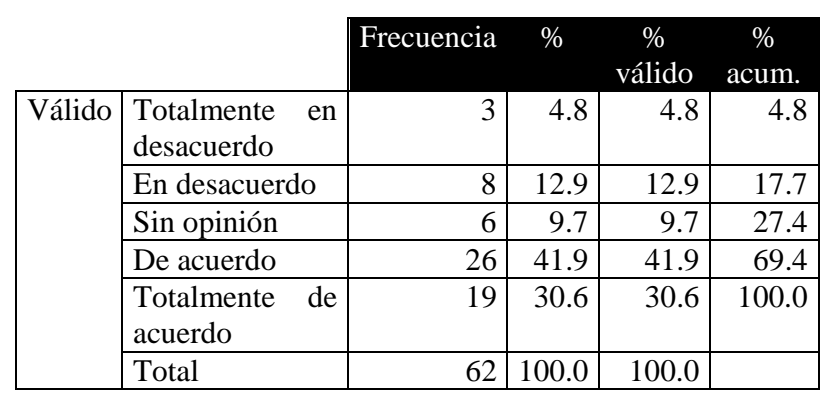

Tabla 8 La motivación en el equipo con eneagrama fue un factor de éxito

Fuente: Elaboración Propia

Para que se logre un resultado positivo en el trabajo en equipo es necesario que todos los miembros de este tengan la motivación suficiente para lograr el éxito. Por ello, se les cuestionó a los integrantes de los equipos sobre como la motivación se ve influenciada por el eneagrama, obteniéndose los siguientes resultados: el $30.6 \%$ se sintió totalmente de acuerdo con la motivación, mientras el $41.9 \%$ está de acuerdo; el 9.7\% no tiene opinión; y, por último, el 12.9 está en desacuerdo y el $4.8 \%$ está en total desacuerdo.

\begin{tabular}{|c|c|c|c|}
\hline & $\mathrm{N}$ & Media & Desv. \\
\hline $\begin{array}{l}\text { Tiempo en que realizó trabajo en } \\
\text { equipo por afinidad }\end{array}$ & 62 & 94.2258 & 11.91558 \\
\hline $\mathrm{N}$ válido (por lista) & 62 & & \\
\hline
\end{tabular}

Tabla 10 Promedio de tiempo de ejecución de tareas en equipos sin eneagrama

Fuente: Elaboración Propia

No queda el menor margen para discutir que uno de los recursos más valiosos de la actualidad es el tiempo en el que se ejecuta correctamente una tarea. En el experimento los equipos de trabajo formados por afinidad tuvieron un tiempo mínimo de 68 segundos y un máximo de 109 segundos, dando una media artimética de 94.22 segundos con un error típico de 11.91 segundos

\begin{tabular}{|l|c|c|c|}
\hline \multicolumn{1}{c|}{} & $\mathrm{N}$ & Media & Desv. \\
\hline $\begin{array}{l}\text { Tiempo en que se realizó trabajo con } \\
\text { eneagrama }\end{array}$ & 61 & 79.5574 & 14.13804 \\
\hline N válido (por lista) & 61 & & \\
\hline
\end{tabular}

Tabla 11 Estadísticos descriptivos de equipos formados con eneagrama

Fuente: Elaboración Propia 
Al tenor de la tabla 10, en la tabla 11 se muestran los resultados de los equipos formados por eneagrama, dando como resultado un tiempo mínimo de 58 segundo y un máximo de 102 segundos, con una media de 79.55 segundos y una desviación estandar de 14.13 segundos

\section{Conclusiones}

Para demostrar estadística y operativamente la funcionalidad de eneagrama es necesario validar el cuestionario, mismo que fue procesado con un paquete de análisis estadístico y se obtuvo una puntuación mayor de 0.80 en la pruebla de Alfa de Crobanch, lo que indica que las respuestas sobre la percepción de eneagrama tienen cierta concordancia y se consideran aceptables las conclusiones que de ellas resulten.

\section{Alfa de Cronbach $\mathrm{N}$ de elementos , 817}

Tabla 12 Estadísticas de fiabilidad Fuente: Elaboración Propia

Como parte del diseño de investigación y para comprobar la correlación entre los sentimiento mostrados y los eneatipos se estableció la siguiente ruta. Cada eneatipo debería de estar más identificado con alguna emoción, para lo cual a la emoción se le asigno un el mismo valor. Es decir, por ejemplo, el eneatipo 1 que se cracteriza por ser visceral y por su estado de alerta debe de indentifacarse en el estudio de su encefalograma con la emoción del estado de alerta, por lo cual se le asigna el mismo número 1. Y cada participante fue evaluado por la emoción predominante durante la ejecución de la tarea asiganda. En la tabla 13 se observa una alta correlación de .912 lo cual indica que si exsite una asociación entre las caracteríticas determinadas por el ejercicio del mapa de eneagrama y las emociones mostradas en el estudio con apoyo de la neurociencia

\begin{tabular}{|l|l|r|r|}
\cline { 2 - 4 } \multicolumn{1}{c|}{} & \multicolumn{3}{|l|}{ ¿Cuál es tu eneatipo? } \\
\hline \multirow{2}{*}{$\begin{array}{l}\text { ¿Cuál es de } \\
\text { eneatipo? }\end{array}$} & $\begin{array}{l}\text { Correlación } \\
\text { Pearson }\end{array}$ & 1 &, $912^{* *}$ \\
\cline { 2 - 4 } & Sig. (bilateral) & &, 000 \\
\cline { 2 - 4 } & $\mathrm{N}$ & 62 & 60 \\
\hline VAR00001 & $\begin{array}{l}\text { Correlación de } \\
\text { Pearson }\end{array}$ &, $912^{* *}$ & 1 \\
\cline { 2 - 4 } & Sig. (bilateral) &, 000 & \\
\cline { 2 - 4 } & $\mathrm{N}$ & 60 & 60 \\
\hline
\end{tabular}

Tabla 13 Correlación entre eneatipo y emociones según neurociencia

Fuente: Elaboración Propia

ISSN 2523-2479

ECORFAN $^{\circledR}$ Todos los derechos reservados
En la tabla 14 se observa que el $78.3 \%$ sí les gustó trabajar con eneagrama y el resto no ven o tienen una percepción positiva sobre el trabajo con eneagrama. $\mathrm{Y}$ en la tabla 15 se demuestra que con un margen de error del $0.3 \%$ que sí existe una diferencia significativa. Entonces, es concluyente que desde la percepción de los participantes sí existe una diferencia favorable en la formación de equipos de trabajo con eneagrama

\begin{tabular}{|c|c|c|c|c|}
\hline & \multicolumn{2}{|c|}{$\begin{array}{c}\text { Te gustó trabajar en } \\
\text { equipo con } \\
\text { eneagrama? }\end{array}$} & \multirow[b]{2}{*}{ Total } \\
\hline & & Sí & No & \\
\hline \multirow{4}{*}{$\begin{array}{l}\text { ¿Habías considerado } \\
\text { trabajar con los } \\
\text { integrantes de tu equipo } \\
\text { según en eneagrama? }\end{array}$} & & 36 & 6 & 42 \\
\hline & & $78.3 \%$ & $37.5 \%$ & $67.7 \%$ \\
\hline & No & 10 & 10 & 20 \\
\hline & & $21.7 \%$ & $62.5 \%$ & $32.3 \%$ \\
\hline \multirow{2}{*}{\multicolumn{2}{|c|}{ Total }} & 46 & 16 & 62 \\
\hline & & $100.0 \%$ & $100.0 \%$ & $100.0 \%$ \\
\hline
\end{tabular}

Tabla 14 Tabla cruzada ¿Habías considerado trabajar con los integrantes de tu equipo según en eneagrama? $\mathrm{Y}_{i \mathrm{Te}}$ gustó trabajar en equipo con eneagrama?

Fuente: Elaboración Propia

\begin{tabular}{|c|c|c|c|}
\hline & Valor & & $\begin{array}{c}\text { Significación } \\
\text { asintótica (bilateral) }\end{array}$ \\
\hline Chi-cuadrado de Pearson & $9,026^{\mathrm{a}}$ & 1 & ,003 \\
\hline Corrección de continuidad $^{\text {b }}$ & 7,257 & 1 & 007 \\
\hline Razón de verosimilitud & 8,631 & 1 & ,003 \\
\hline \multicolumn{4}{|l|}{ Prueba exacta de Fisher } \\
\hline Asociación lineal por lineal & 8,880 & 1 & ,003 \\
\hline $\mathrm{N}$ de casos válidos & 62 & & \\
\hline
\end{tabular}

Tabla 15 Prueba de Chi cuadrado

Fuente: Elaboración Propia

La efectividad del eneagrama para formar equipos de trabajo queda de manifiesto al hacer el comparativo de medias (Tabla 16), donde con un margen de error $(0 \%)$ aceptable menor $5 \%$ se puede afirmar que los equipos de trabajo con eneagrama realizar en menor tiempo las tareas asignadas, $\mathrm{y}$ al ser el tiempo un factor determinante en el éxito de los equipos de trabajo se concluye que son más exitosos que los equipos formados por afinidad

Grupo N Media \begin{tabular}{|l|l|l|l|}
\hline Equipo & Con eneagrama & 62 & 79.2742 \\
\cline { 2 - 4 }
\end{tabular} \begin{tabular}{|l|l|l|}
\hline Sin eneagrama & 62 & 94.2258 \\
\hline
\end{tabular}

\begin{tabular}{|l|c|c|c|c|}
\hline \multicolumn{5}{|c|}{ Prueba de Levene de igualdad de varianzas } \\
\hline \multicolumn{1}{|c|}{$\mathrm{F}$} & Sig. & $\mathrm{t}$ & $\mathrm{gl}$ & $\begin{array}{c}\text { Sig. } \\
\text { bilateral }\end{array}$ \\
\hline $\begin{array}{l}\text { Se asumen varianzas } \\
\text { iguales }\end{array}$ & 3.750 & .050 & 119 & .000 \\
\hline $\begin{array}{l}\text { No se asumen varianzas } \\
\text { iguales }\end{array}$ & & & 116.436 & .000 \\
\hline
\end{tabular}

Tabla 16 Prueba de medias para muestras relacionadas Fuente: Elaboración Propia

LINAREZ-PLACENCIA, Gildardo, ESPINOZA-CASTELO, Luz Maria y PIMENTEL-FÉLIX, Ana Fabiola. Neurociencia y eneagrama: Reconfigurando los equipos de trabajo. Revista de Pedagogía Crítica. 2019 
Las conclusiones son contudentes en todas las pruebas estadísticas elaboradas para el diseño cuasiexperimental propuesto, y se resumen en tres puntos:

1. Hay evidencia signficativa demostrada que los equipos de enegrama son más eficientes porque realizan las tareas en un menor tiempo al existir coordinación, liderazgo, organización y motivación.

2. La percepción de los participantes es positiva sobre los mismos elementos mencionados en el punto anterior, se demostró estadísticamente no quedando duda alguna.

3. Con ayuda de la neuorociencia se detectaron que las emociones y sentimientos según los eneatipos son reales y corresponden generalmente según la técnica del eneagrama.

4. Recomendaciones

Se recomienda que las organizaciones tomen en cuenta el eneagrama como una técnica válida para la formación de equipos de trabajos. Asimismo, en la contratación de nuevo personal deben de atender a los eneatipos que ya tienen dentro de la organización para que pueden sacar mejor provecho del capital humano.

También es importante que las organizaciones vean hacia su interior, para que su capital humano pueda conocerse, para así tratar de establecer procesos de mejora continua que eleven su potencialidades y desarrollen ampliamente sus áreas de oportunidad.

\section{Referencias}

Aiger, M., Palacín, M., \& Cornejo, J.-M. (2013). La señal electrodérmica mediante Sociograph: Metodología para medir la actividad grupal. Revista de Psicología Social, 28(3), 333-347.

Barthelemy, H. R., \& Rodriguez, V. G. (2016). Título: observaciones recientes acerca del aporte de las neurociencias en la práctica y el estudio de la administración. Recuperado de http://www.adenag.org.ar/uploads/congresos/na cionales/3.8_BARTHELEMY_RODRIGUEZ_. pdf

Beesing, M., Nogosek, R. J., \& O’Leary, P. H. (1992). El eneagrama: Un camino hacia el autodescubrimiento (Vol. 154). Narcea Ediciones.
Benito, S. M. (2011). Neuromarketing: Tecnologías, Mercado y Retos/Neuromarketing: Technologies, Market and Challenges. Pensar la publicidad, 5(2), 19-42.

Braidot, D. N. P. (2013). Neuromanagement y Neuroliderazgo. Cómo se aplican los avances de las neurociencias a la conducción y gestión de organizaciones. Ciencias Administrativas, O(2). Recuperado

de http://old.revistas.unlp.edu.ar/CADM/article/vie w/706

Campos, R. F., \& Álvarez, L. G. (2019). ?’ Por qué la neurociencia debería ser parte de la formación inicial docente? Synergies Chili, (15), $45-125$.

Castro Castillo, P. A. (2019). Impacto de los líderes de talento humano en la organización.

Delgado, P. (2014). Eneagrama. Una herramienta en la gestión del factor humano. I Jornada Binacional de Investigación de la URBE (CICAG), 39.

Fernández, L. M. (2010). Los 9 tipos de personalidad del eneagrama.

Hernández, M. W. H., Armijo, C. E. G., \& Sánchez, R. E. R. (2019). '’ Qué es el ser humano o la persona? La educación o la formación integral del ser humano. HELIOS, 2(2).

López Muñoz, F., Rubio, G., Molina, J. D., García García, P., Álamo, C., \& Santo Domingo, J. (2008). Cajal y la Psiquiatría Biológica. El legado psiquiátrico de Ramón y Cajal (una teoría y una escuela). Arch. psiquiatr, 50-79.

López-Cantos, F., \& Millán Yeste, J. (2018). La difusión de discursos pseudocientíficos en la radio pública española. El programa Complementarios de RNE-Radio 5.

Mascareño, A. (2018). De la crisis a las transiciones críticas en sistemas complejos: Hacia una actualización de la teoría de sistemas sociales. Theorein. Revista de Ciencias Sociales., 2(1), 109-143.

Molina, R., Israel, R., \& Ampudia Sjogreen, D. E. (2018). Factores de competitividad empresarial en el sector comercial. 
Ramírez Villanueva, A. (2018). Estudio sobre la personalidad de los influencers en Instagram.

Requena, R. A. (2005). El Neuromarketing. Una visión diferente para entender al cliente. Perspectivas, 8(2), 83-97.

Riso, D. R. (1993). Tipos de personalidad: El eneagrama para descubrirse a sí mismo. Editorial Cuatro Vientos.

Rúas-Araújo, J., Punín Larrea, M. I., Gómez Alvarado, H., Cuesta-Morales, P., \& Ratté, S. (2015). Neurociencias aplicadas al análisis de la percepción: Corazón y emoción ante el Himno de Ecuador. Revista Latina de Comunicación Social, (70).

Vargas, A. (2015). Eneagrama (Nueva edición):?`Quién soy? Alamah.

Villegas, N. C. P., \& Sánchez, O. R. P. (2018). Estrategias para el desarrollo de competencias sociales y emocionales como herramienta en la gestión del desempeño docente. Red de Investigación Educativa, 10(1), 1-14. 